\title{
CURVATURE CHANGES IN BIOLOGICAL DYNAMICS
}

\author{
Arturo Tozzi (corresponding Author), \\ Center for Nonlinear Science, University of North Texas \\ 1155 Union Circle, \#311427 Denton, TX 76203-5017 USA \\ tozziarturo@libero.it \\ Arturo.Tozzi@unt.edu
}

Geometry deals both with analogical thinking and physical/biological observables. Naïve, common-sense descriptions of objects' shapes and systems' trajectories in geometric phase spaces may help experimental investigation. For example, very different biological dynamics, as the developmental growth patterns of the oldest known animal (the extinct Dickinsonia) and the human brain electric oscillations, display a striking analogy: when encompassed in abstract geometric spaces, their paths describe the same changes in curvature: from convex, to flat, to concave and vice versa. This dynamical behavior, anticipated by Nicholas de Cusa in his analogical account of "coincidentia oppositorum" (1440), helps to describe widespread biological paths in the manageable terms of concave, flat and convex curves on donut-like structures. Every trajectory taking place on such toroidal manifolds can be located, through a topological technique called Hopf fibration, into a four-dimensional space. We discuss how the correlation between Hopf fibration and Navier-Stokes equations allows us to treat the above-mentioned biological and neuroscientific curved paths in terms of flows taking place into a viscous fluid medium that can be experimentally assessed and quantified.

Keywords: lines; brain; topology; curvature; Dickinsonia; fMRI.

Analogy, i.e., the common-sense identity of relation between apparently unrelated objects or features, is the ordinary way for human mental activity to cope with everyday life (Sowa and Majumdar, 2003). Through analogy, our brain tries to make sense of the unpredictable features of the ceaseless environmental inputs (Hofstadter and Sander, 2011). Despite its scarce scientific power, analogy paves the way to novel perspectives that may be propaedeutic to build the proper experimental settings with the hallmarks of scientific activity. To provide a few examples, pairwise comparison is framed on the quantitative analysis of two "things" that display matching description (Koczkodaj et al., 2017). Such matching description concerns similarity between two observables, but the demarcation among objects and things could be somewhat arbitrary, because our mind tends to exclude the continuity among the hidden structures of the world. We tend to draw a line of separation among things that we judge different, arbitrarily excluding or including issues in our description, in order to achieve positive demarcations that allow a pragmatic treatment of the world, based on regularity and uniformity (Popkin and Maia Neto, 2007; Autrecourt, 1340). The same holds for set theory: the description of sets is not always quantitative. Indeed, in touch with set theory, our mind, equipped with logic weapons, tends to split the entire world in different, arbitrary, fictious subsets that do not really stand, in terms of quantum dynamics, for different things. Further, in topology, a closed Jordan curve separates fairly subjective and abstract external and internal surfaces (Munkres 2000; Ghrist 2014; Peters 2017). In turn, classical logic, based on the principles of identity, non-contradiction and so on, uses categories able to encompass the concept of "identical". However, logic does not describe the world, rather the human interpretation of the world: indeed, identity might stand not just for sameness, but also for the analogical "belonging to" (Heidegger 1957; Tozzi and Peters, 2018). The same scientific concept of "observable" is based on the choice of variables in a limited experimental setting: scientists, putting aside most of them, focus their efforts on just a few features: e.g., elementary particles are defined just in terms of three experimental observables, i.e., charge, spin and mass, letting aside, for practical purposes, less "useful" features.

However, despite the above-mentioned limitations, analogical thinking allows scientist to achieve the preliminary "illumination" which later will become an experiment to analyze through the powerful scientific tools of probability and mathematical weapons (Wigner, 1960). To make an example, the analogical concept of changes from positive to flat curvatures for the description of nature dates back to the philosophical efforts of Nicholas de Cusa's "coincidentia 
oppositorum" (1440). His analogical framework, deprived of its old-fashioned, untenable metaphysical framework, can be used to build a modern description of biological dynamics. Focusing on de Cusa's account and on curvature changes in biological paths, we will describe how novel approaches, based on the intrinsic relationship between analogical and geometrical interpretation of the world, allow quantitative comparison of living systems' observables, depicted as curved lines endowed in positive, negative- or zero- curvature manifolds.

\section{THE USE OF GEOMETRIC ANALOGIES: THE OLD AND THE NEW}

In this Section we will show how is it feasible to use the analogy between geometric drawings and physical/biological observables in order to reach scientific knowledge. We will provide the example of curvatures in phase spaces; indeed, unqualified manifold curvature is often unable to assess or measure physical observables: when we set out to investigate the manifolds where biological dynamics take place, we must leap beyond simple physical concepts and use mathematics. Inspired by Nicholas de Cusa' treatment of infinity (de Cusa, 1440; Tozzi and Peters, 2017), here we show how analogy and geometric signs ought to be used in undertaking the manifolds underlying physical/biological paths. If we want to assess physical/biological measurements leading to a description of our world, we need at first to consider mathematical figures and topological manifolds, together with their features and relations. Next, we must apply these relations in a projective way. Thirdly, we must thereafter, in a still more highly transformed way, apply the relations of these infinite figures to the general concept of mathematical curvature, which is altogether independent even of all figures and manifolds.

De Cusa's account: infinity as a zero-curvature, straight line in geometry. De Cusa depicts the mathematical infinite as a straight line. He maintains that, if there were an infinite line, it would be a straight one. This means that hypothetical infinite triangles, circles or spheres can be described in terms of infinite lines, because they display infinite sides. De Cusa proceeds as follows: the circle's diameter (which, in modern terms is a positive-curvature, convex manifold) is a straight line and its circumference is a curved line greater than the diameter. Therefore, if the curved line (with positive-curvature) becomes less curved in proportion to the increased circle's circumference, then the maximum circle's circumference, which cannot be greater, is minimally curved and maximally straight. Indeed, in Figure 1A, the convex arcs of the larger circle are less curved than the smaller ones. Therefore, the straight line will be the arc of the maximum circle, which cannot be greater. An infinite line is necessarily the straightest; and to it no curvature is opposed. In the same way, every manifold with positive curvature, such as, e.g., a triangle, or a circumference, or a sphere, can be described in terms of an infinite line standing for a maximum triangle, or a maximum circle, or a maximum sphere. We know, De Cusa argues, that a line finite in length can be longer and straighter; and that the maximum line is the longest and straightest. If these figures are describable by a finite line, and if an infinite line is all the things with respect to which a finite line is in infinity, then it follows, according to de Cusa, that an infinite line stands also for a triangle, a circle, and a sphere. The philosopher, displaying analogical thinking, says: how is it possible that an infinite line is a side of a triangle? Since any two sides of any triangle cannot, if conjoined, be shorter than the third, this means that, in the case of a triangle whose one side is infinite, the other two sides are not shorter, i.e., they are both infinite. And because each part of what is infinite is infinite, for any triangle whose one side is infinite, the other sides must also be infinite. Since there cannot be more than one infinite thing, an infinite triangle cannot be composed of a plurality of lines, even though it is the greatest and simplest triangle. And because it is a triangle-something which it cannot be without three lines-it will be necessary that the one infinite line be three lines, and that the three lines be one most simple line. Similarly, the analogy holds also for the three angles. Nor will this maximum triangle be composed of sides and angles; rather, the infinite line and angle are one and the same thing, so that the line is the angle, because the triangle is the line. The larger the one angle, the smaller are the other two. Now, any one angle can be increased almost but not completely up to the size of two right angles. According to de Cusa, it will be obvious that the triangle has one angle which is three angles and that the three angles are one. In the same manner, we can state that a triangle is a line. For any two sides of a quantitative triangle are, if conjoined, as much longer than the third side as the angle which they form is smaller than two right angles. Hence, the larger the angle, the less the lines and the smaller its surface. Hereby it is evident that an infinite line is a maximum triangle.

Next, using the same explanatory arguments, de Cusa argues that the maximum triangle is also a circle and a sphere, because an infinite triangle is also an infinite circle. Moreover, that an infinite line is a sphere becomes very obvious 
by applying the same reasoning. Therefore, according to the philosopher, an infinite line is actually and infinitely all that which is in the possibility of every finite line and manifold. Hence, he highlights a noteworthy speculative consideration, based on analogy between abstract geometric lines and real things: infinity is correlated with finite manifolds, and these manifolds may change in curvature. Here comes into play the concept of "coincidentia oppositorum": because infinite curvature is infinite straightness, this means that an infinite manifold can be described in opposite terms: it is not a thing and is not any other thing; is not here and is not there; is unqualifiedly free from all things and is beyond all things; is above the negation of all things.

Updating analogy: de Cusa 3.0. In modern terms, de Cusa states that manifolds may be tackled in terms of convex curves progressively approaching a straight line with zero-curvature, i.e., the line that the philosopher calls "infinity". De Cusa's description, in the light of current advances in math, can be extended to encompass not just convex curves tending towards straight ones, but also concave ones (Figure 1B). Indeed, non-Euclidean geometries, unknown at de Cusa's times, cope with manifolds with any curvature (either positive-, negative- or zero-curvatures). Therefore, biological dynamics (such as, for example, neural dynamics) can be thought as taking place in phase space manifolds which can be flat, concave or convex (Borsuk 1969; Tozzi et al., 2018a). This improved de Cusa's framework, dealing with different-curvature manifolds' dynamics, can be used to mathematically describe and treat biological systems. As we will see later, convex or concave trajectories can be projected to straight ones and vice versa, through wellknown transport procedures (Frankel, 2011; Sengupta et al., 2016).

Hints of the de Cusa's account of spheres tending towards infinity can be found also in modern topology. The socalled Hopf fibration describes a hypersphere (i.e., a four-dimensional structure with positive-curvature) in terms of the circles embedded in an ordinary three-dimensional sphere. The stereographic projection of Hopf fibrations generates a peculiar structure on a four-dimensional hypersphere, in which the space is filled with nested tori. Each fiber maps to a circle in space: it is noteworthy that one of the fibers projecting to a circle is a line, which is topologically defined, with a terminology that closely resembles de Cusa's terms, a "circle through infinity" (Figure 1C). Furthermore, de Cusa's concept of "coincidentia oppositorum" develops an even older belief, i.e., that microcosmos mirrors macrocosmos, so that all the cosmic things are intertwined at every scale of observation. In touch with this ancient holistic view of the Universe, a striking analogy can be drawn with a successful contemporary scientific issue: the concepts of (spatial) fractals and (temporal) power laws, which describe our world as intermingled series of self-repetitive structures, ranging from micro-to macro-scales (Mandelbrot 1967; Fetterhoff et al., 2014). 


\section{A}

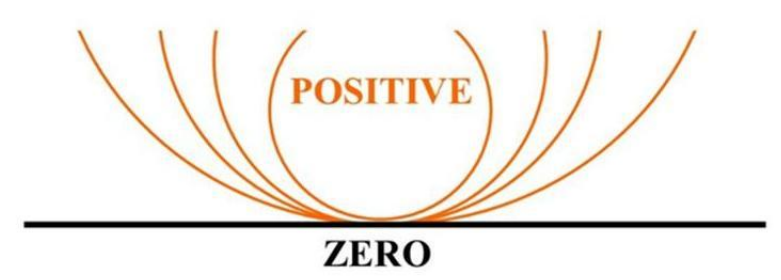

B

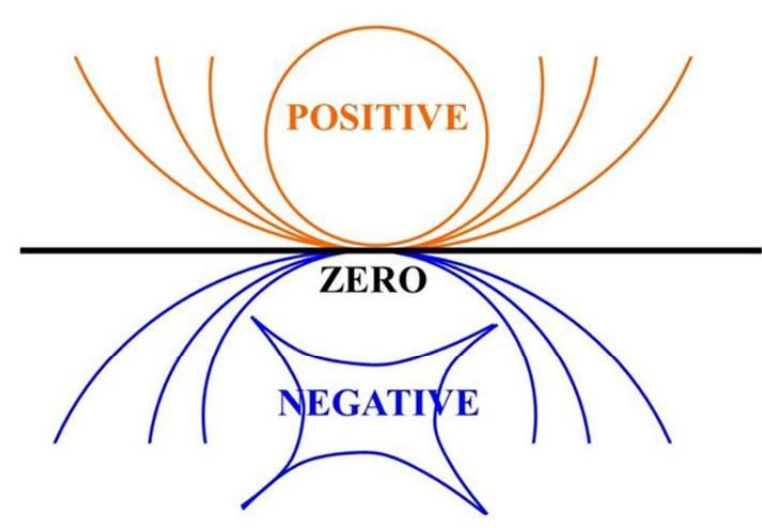

C

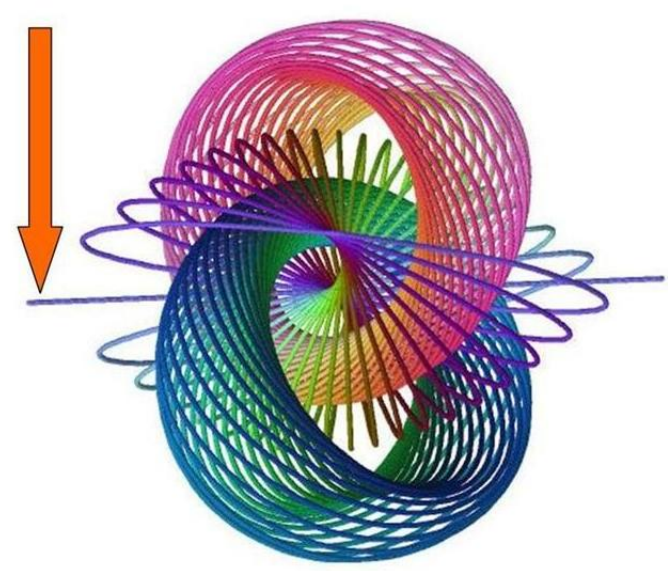

Figure 1A: de Cusa's account of convex curves of increasing radius, until a straight line is achieved. Figure 1B: in modern terms, different curvatures stand for changes in manifolds' structure. A physical/biological system can be described by progressively increasing curves on positive- or negative-curvature manifolds, until the the occurrence of infinity (zero-curvature horizon) is reached. Figure 1C: Hopf fibration and its relationships with de Cusa's lines. The three-dimensional figure illustrated here is endowed in a hidden four-dimensional space with positive curvature. The convex curves display different curvatures, depending on the different ray's length of every circle. The circle with 
the highest curvature and infinite radius can be depicted as a straight line (red arrow), in touch with de Cusa's ancient account. Modified from: Johnson (2018).

\section{BIOLOGY COMES INTO PLAY}

The enigmatic Dickinsonia is an extinct lineage that does not fit within known animal phyla. Dated about 571-541 million years ago, it is a marine genus of fossils belonging to the early communities of Ediacara Biota, i.e., the oldest fossil evidence for the appearance of animals (Ivantsov 2007). Recently assigned to the animal kingdom (Bobrovskiy et al., 2018), the mobile Dickinsonia lacks convincing evidence of mouth, gut and anus and possibly fed via external absorption and digestion of organic matter through its ventral surface. The developmental patterns of Dickinsonia's isometric and indeterminate growth have been recently studied by Evans et al. (2017), Gold et al. (2015) and Hoekzema et al. (2017). Dickinsonia modules grew by terminal addition and module inflation in a highly complex manner. As illustrated in Figure 2A, the approximate trend in Dickinsonia's module shape change is the following: posterior modules run straight from the midline to the outer edge at an angle less than 90 degrees, giving rise to a " $v$ " (i.e., a convex, positive-curvature) shape (Evans et al., 2017). From the posterior towards the middle of the long axis, the angle increases, until it becomes perpendicular (i.e., zero curvature) to the midline. From this point to the anterior, a bending of anterior modules occurs, so that they are still approximately perpendicular where they intersect the midline, but become roughly parallel to the mid-line closer to the outer edge, forming "u" shaped (i.e., a concave, negative curvature) modules. Therefore, the growth of this puzzling animal clearly resembles the changes in curvature patterns described in the previous paragraphs, e.g., modifications of its outer biopolymer in terms of convex, concave and flat lines.

The same curvature patterns can be found in a very different scientific discipline, i.e., in neuroscience. Peters et al. (2016) recently introduced a novel image-analysis technique for the measurement of information level in fMRI (functional Magnetic Resonance Imaging) neural data sets, based on image subdivision in small polygons equipped with different entropic content. This approach facilitates the objective detection of hidden temporal patterns of entropy/information in zones of fMRI images generally not considered. This method, which befits the geometric character of fMRIs, was able to detect the presence of a fourth dimension's distinctive hallmarks in a temporal sequence of two-dimensional images taken during spontaneous brain activity (see also: Tozzi and Peters, 2016). The trajectories described by these temporal patterns closely resembles a wave, which front-line is characterized by the curvatures' modifications described in this paper and anticipated by de Cusa. 
A

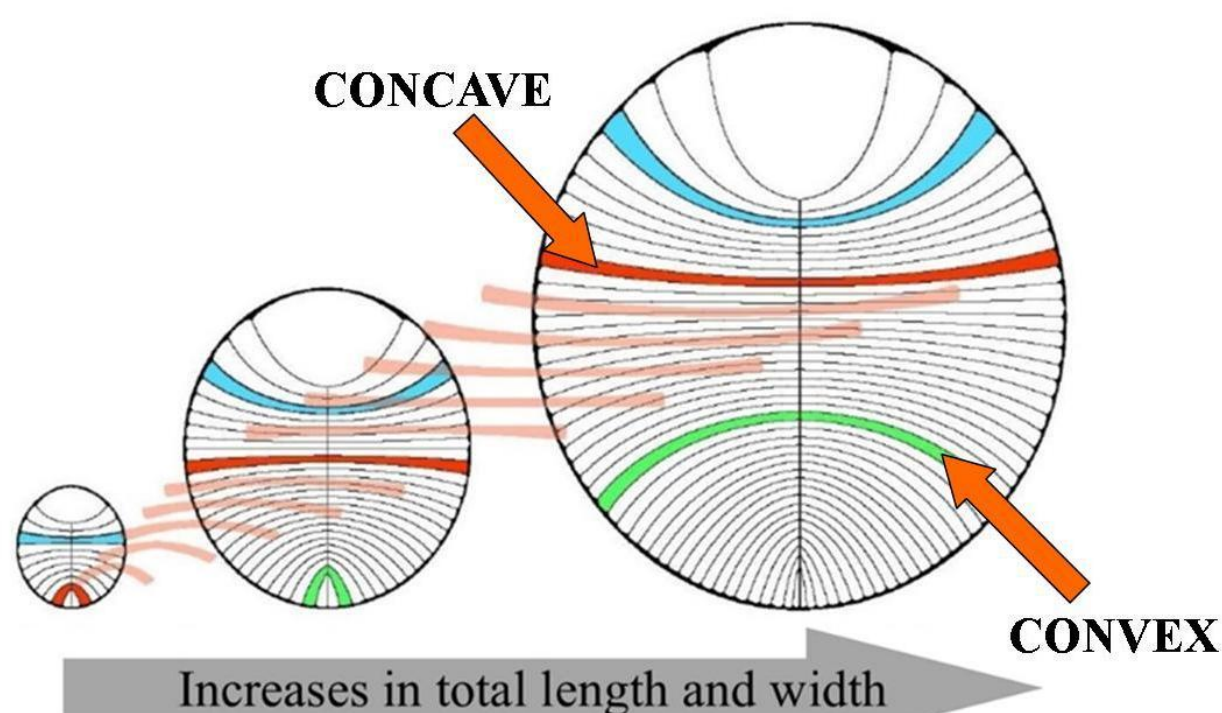

B

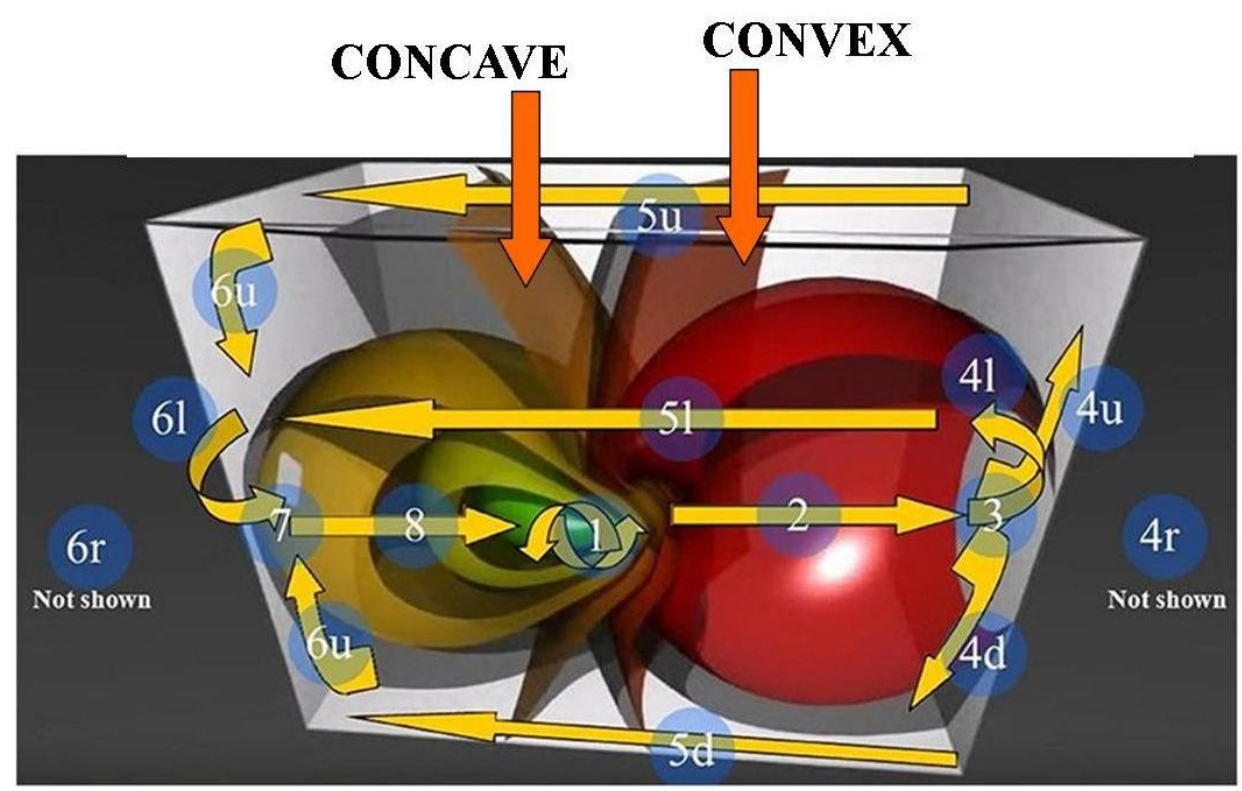

Figure 2. Biological examples of changes in manifold curvatures. A: Sketch of Dickinsonia growth. Changes in modules shape and size occur with increase in total length and width. From: Evans et al. (2017). Figure 2B. Multidimensional activity of brain dynamics, according to Peters et al. (2016). The Figure illustrates a threedimensional stereographic projection of the "toroidal parallels" of a four-dimensional hypersphere. The orange arrows depict the trajectories followed by the quaternionic movements of a four-dimensional Clifford torus projected onto the surface of its internal three-dimensional space. The circled numbers describe trajectories which conventionally 
start from the point 1 . The letters $\mathrm{u}, \mathrm{d}, \mathrm{r}, 1$ stand respectively for the upper, down, right, and the left trajectories on the surfaces of the three-dimensional structure. In our picture, the spheres on the right grow in diameter, forming a circle of increasing circumference on the right surface of the three-dimensional space. Conversely, on the opposite left side, the spheres shrink and give rise to a circle of decreasing circumference on the left surface of the three-dimensional space. Wave fronts (red arrows on the top of the Figure) closely resemble de Cusa's spheres with progressive modifications in radius length. In sum, convex lines (standing for the electric spikes' wave fronts experimentally detectable in the human brain) become at first flat, then concave. Modified from: Peters et al. (2016) and: https://www.youtube.com/watch?v=QlcSlTmc0Ts.

\section{HOW TO COPE WITH MANIFOLDS OF DIFFERENT CURVATURES: THE PROCEDURES}

In this Section, we will answer the crucial question: once established relationships among curvature changes in biological systems, how could we use them in order to assess scientific issues and quantify natural phenomena?

Curvatures changes on toroidal manifolds: Hopf fibrations and Navier-Stokes equations come into play. Countless biological paths, including the ones described above (Dickinsonia growth and nervous dynamics), can be tackled in terms of paths of different curvatures embedded in a three-dimensional phase space. The relationships among the positive-, zero- and negative-curvature manifolds can be defined through a standardized procedure (Figure 3). At first, real-world trajectories (Figure 3A) can be transported to the opposite convex and concave sides of a twodimensional torus (Figure 3B). Manifold projections can be achieved through already-available methodologies of vectorial and tensorial transport: such as Ehresmann connections (Tozzi et al., 2018b) or parallel transport on Riemannian manifolds (Sengupta et al., 2017). These procedures allow us to achieve a manageable representation of countless biological trajectories, because paths on toruses are well-known and already described. Indeed, many of their features have been mathematically assessed, such as modular wavelets, admissibility and frame conditions, lifting admissible functions, transformations and dilations, reconstruction formulas, required Lie groups and extra modular groups, natural tensor products (Calixto et al., 2015; Wang et al., 2015).

Once achieved a description of different curvatures on a donut-like structure, we are allowed to go further. A topological technique, called Hopf fibration (Hopf, 2001), allows us to project lines from the two-dimensional torus onto a four-dimensional manifold. The procedure is illustrated in Figures 3C-3D. Every circle on the torus is projected to a curve embedded in a three-dimensional sphere (Figure 3C). Every point of the three-dimensional sphere's surface matches a single circular trajectory on a four-dimensional hypersphere, in an organized and symmetric fashion (Figure 3D). In mathematical terms, the circular trajectories on the 3-sphere match the compact, simply connected symplectic Lie group $S p(1)$ equipped with quaternionic $1 \times 1$ unitary matrices.

The question here is: once we "transmute" biological paths into four-dimensional trajectories through a Hopf fibration, what do we achieve? What for? The answer is straightforward, if we take into account that the Hopf fibration is able to describe, among various issues, also the dynamics of the Navier-Stokes equations (Vankerschaver and Leok, 2014). These equations' solution is the flow velocity field, defined at every point in a region of space and an interval of time (White 2006). Different from classical mechanics, Navier-Stokes equations study velocity instead of position (Gorban and Karlin, 2016); this means that they are very useful for the study of viscous fluids. Therefore, our proposal to treat biological dynamics in terms of Hopf fibrations allows to tackle them in the language of the Navier-Stokes equations, as if living paths were flows taking place in a fluid medium. This means that Dickinsonia's development and nervous dynamics of the human brain become more manageable phenomena of fluid dynamics. 


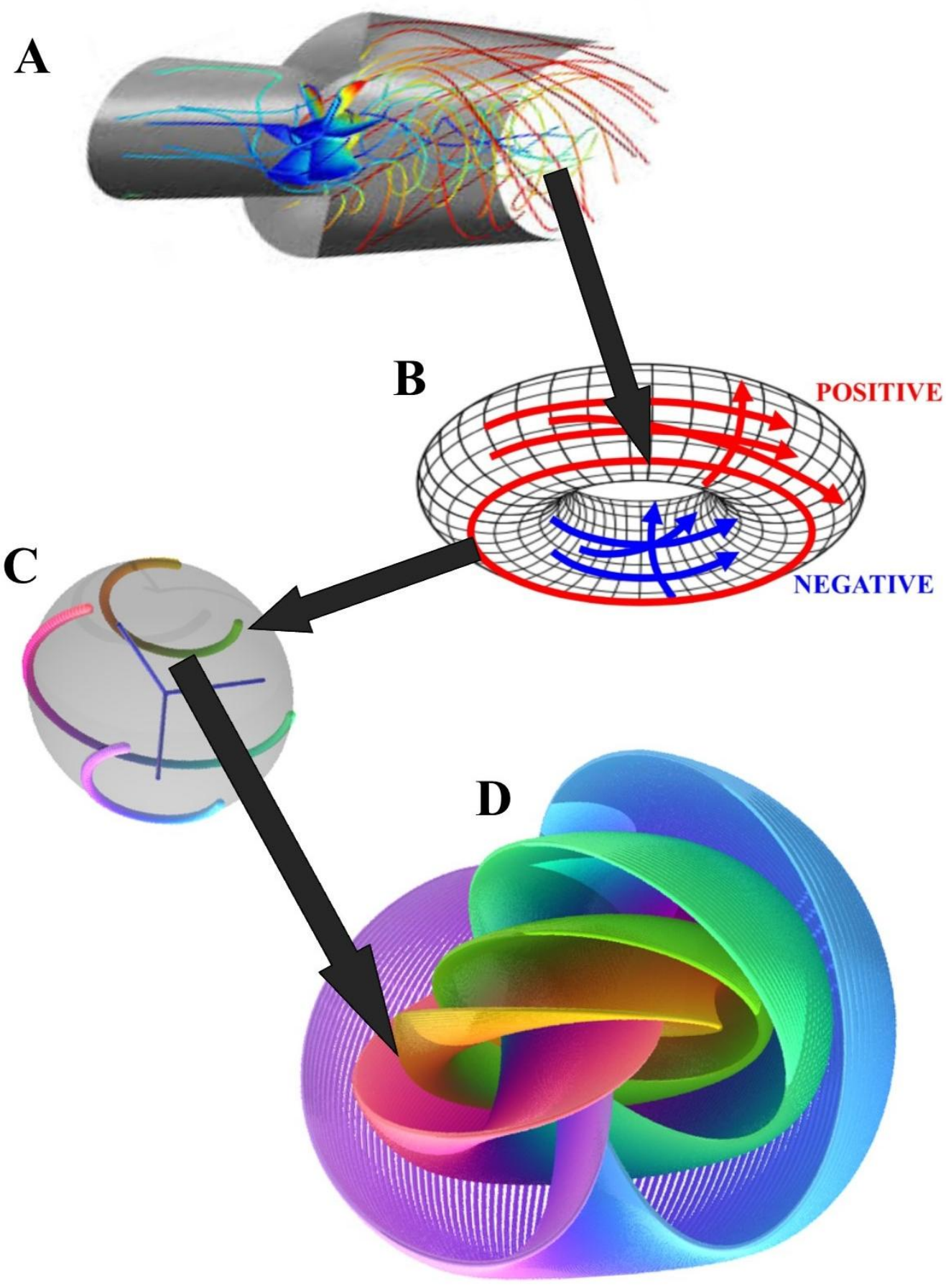

Figure 3. Widespread physical and biological paths of every kind of curvature (Figure 3A), can be described in terms of concave, flat and convex paths on a torus (Figure 3B). Therefore, by placing physical or biological observables on a toroidal manifold, we achieve a description of trajectories on a donut-like phase space. Every one of the achieved trajectories on the torus can be projected to the two-dimensional circular trajectories taking place on a threedimensional sphere (Figure 3B). In turn, these two-dimensional trajectories, standing for the curved lines originally 
described by de Cusa, can be projected to a four-dimensional space with positive curvature through Hopf projections (Figure 3B).

Modified from: CFD Moduule. https://www.technic.com.au/products/cfd/ (Retrieved 2018), and Johnson (2018).

\section{CONCLUSIONS: A MENTAL-FRAMED ANALOGICAL WORLD}

In this paper, we aimed to show that geometry can be used as an analogical tool that our mind employs to cope with environmental observables. We provided examples which elucidate how analogical thinking might be useful in developing novel experimental procedures and testable hypotheses in a real scientific setup. At first, we described the early de Cusa's formulation of "coincidentia oppositourum" in terms of curvature changes. Then, we "updated" his account, in the light of the current topological concepts of concave, flat and convex manifolds. We assessed real world phenomena and showed that the paths we described are widely displayed by biological dynamics. We emphasized the advantages of such approaches in the quantitative assessment of the dynamics of living beings, depicted in terms of trajectories and wave-fronts taking place on phase spaces equipped with changing curvatures. This allows us to mathematically describe biological functional trajectories in terms of fluid dynamics on a computationally manageable donut-like structure. Our main result is that dissimilar biological paths, which may be apparently linked just by analogies, can be described in the same operational terms. Indeed, using the proper topological treatment, either curved or straight paths can be assessed through Navier-Stokes equations. The latter are very useful for the study of viscous fluids, including the very intricated turbulent convective flows characterized by nonlinearity and chaotic behavior. However, the numerical solution of the Navier-Stokes equations for turbulent flows is extremely difficult and computationally expensive, due to the significantly different mixing-length scales involved in the phenomenon (Holdeman 2010). Our account might help to simplify the approach to Navier-Stokes equations in nonlinear flows: once every turbulent flow is projected to the same toroidal structure, it would be easier to calculate their paths, via the suggested procedure of Hopf fibrations.

Once achieved that analogical thinking can be successful in tackling scientific issues, our next goal is to deliver a psychological explanation for our innate mental ability to use geometry and analogy in describing the world. In other words, our brain tends to believe that geometric analogies are a faithful mirror of the environment. Recent neuroscientific findings could explain why human mind seems to recognize Euclidean points, lines, surfaces, volumes, despite the fact that they do not exist in the real world. Over the last few years, studies of receptive field properties in several mammalian visual brain structures, such as the lateral geniculate nucleus and the primary sensitive cortex, have suggested the existence of cell classes with unique functional response properties (Van Hooser et al., 2005; Viswanathan and Nieder, 2017). For example, the cells of the cortical visual area V1 display basic receptive field properties, such as orientation selectivity, direction selectivity, bar length and end-stopping (Duffy and Hubel, 2007). Similar to the primary visual cortex, orientation sensitivity was closely reflected by the receptive field properties also in extracortical structures, such as the lateral geniculate nucleus ( $\mathrm{Li}$ et al., 2018). Therefore, recent experimental suggestions point towards our brains as equipped with neurons, located in cortical and extra-cortical areas, that selectively fire when our sight perceives angles, surfaces, lines, contours. Such angles, surfaces, lines, contours do not exist in the real world, provided that the objects' borders cannot be as well sharply delimited as suggested by Euclidean geometry. Since many years, there is the problem in weighting and precision metrology to consider where the mass surface ends, because there are always, without solution of continuity, "layers" of air molecules more or less interacting with the "solid", part of which does influence the "weighted mass" also in the most accurate measurements. "The non-uniqueness in these cases comes from thickness of the adherent interface layer(s), having an effect different from zero on the actual dimensions of the object, i.e., adding additional mass to the object with possibly timedependent variability" (Pavese and Charki, 2016).

Our perception is perhaps an evolutive mechanism that allows us to cope with our external, rather visual, world, in order to avoid dangers and harms. The detection by sight of geometrical issues in the environment, such as points, lines, contours and so on, stands for a sort of species-specific internal map, an innate interpretation of the physical reality. We are rather firmly wired, if not actually hardwired, to think of the abstractions represented by such programs 
as realities. The whole idea in biology is after all to respond to survival situations quickly and effectively, rather than to question or contemplate their deeper nature. In Karl Friston's terms (2010), the brain is an inferential machine that compares the real world with an internal map, pre-existent in our mind. Therefore, it might be suggested that what we see, rather than being real, are framed on analogies. The same holds for other animals, such as, e.g., an ant equipped with olfactive-tactile perceptions rather than our visual ones, or a bat (Nagel, 1974). Some response properties, such as linearity of spatial summation, contrast gain, dependence of receptive field size on eccentricity and interlaced arrangement of cortical neurons, vary from species to species (Van Hooser et al., 2005; Mazade and Alonso, 2017). To make an example, despite the fact that a squirrel has greater visual acuity and a physically larger V1 than other mammals that have orientation maps in V1, this animal is not provided of such maps (Van Hooser et al., 2005). In turn, electrophysiological recordings in awake and anesthetized turtles reveal that their dorsal cortex is sensitive to the spatial structure of natural images, and their receptive fields are not entirely uniform across space (Fournier et al., 2018). The mapping and representation of visual space in turtle's three-layered cortex differ from those found in mammalian primary visual cortex. This means that, contrary to the mammalian primary visual cortex, the turtle dorsal cortex performs a global, rather than local, analysis of the visual scene (Fournier et al., 2018). In animals different from us, a clear retinotopic mapping of thalamocortical projections is not detectable and spatial information can be found both at the single-neuron and population scales. A recent study points towards the hypothesis that our sensations and perceptions (and therefore our world) depend on the activity of our cortex. Armenta Salas et al. (2018) showed that intracortical micro-stimulation in primary somatosensory cortex (S1) is a method of inducing discriminable artificial sensation. Replicable elicitations of sensations in both the cutaneous and proprioceptive modalities were reported in a tetraplegic human with microelectrode arrays implanted in S1. This means that direct activation of the cortex produces naturalistic percepts that closely mimic the body's natural physiological capabilities. And it is achieved through analogical mechanisms.

\section{REFERENCES}

1) Armenta Salas M, Bashford L, Kellis S, Jafari M, Jo H et al. 2018. Proprioceptive and cutaneous sensations in humans elicited by intracortical microstimulation. Elife. 2018 Apr 10;7. pii: e32904. doi: 10.7554/eLife.32904.

2) Autrecourt, Nicholas of. About 1340. The Universal Treatise. Marquette University Press, Milwaukee, Wisconsin, 1971

3) Bobrovskiy, Ilya; Hope, Janet M.; Ivantsov, Andrey; Nettersheim, Benjamin J.; Hallmann, Christian; Brocks, Jochen J. (20 September 2018). "Ancient steroids establish the Ediacaran fossil Dickinsonia as one of the earliest animals". Science. 361 (6408): 1246-1249. doi:10.1126/science.aat7228

4) Borsuk K. 1969. Fundamental retracts and extensions of fundamental sequences, Fundamenta Mathematicae $1: 55-85$.

5) Calix to M, Guerrero J, Roşca D. 2015. Wavelet transform on the torus: A group theoretical approach. Applied and Computational Harmonic Analysis, 38 (1): 32-49. https://doi.org/10.1016/j.acha.2014.03.001.

6) de Cusa N. 1440. De docta ignorantia. English translation in Bond, H. Lawrence (ed.), Nicholas of Cusa: Selected Spiritual Writings, Classics of Western Spirituality. New York: Paulist Press, 1997.

7) Duffy KR, Hubel DH. 2007. Receptive field properties of neurons in the primary visual cortex under photopic and scotopic lighting conditions. Vision Research. Volume 47, Issue 19, September 2007, Pages 2569-2574.

8) Euclid, 300 BCE. Elements of Geometry, trans. by R. Fizpatrick, farside.ph.utexas.edu/Books/Euclid/Elements.pdf.

9) Evans SD, Droser ML, Gehling JG. 2017. Highly regulated growth and development of the Ediacara macrofossil Dickinsonia costata. Plos ONE. https://doi.org/10.1371/journal.pone.0176874.

10) Fetterhoff D, Opris I, Simpson SL, Deadwyler SA, Hampson RE, Kraft RA (2014) Multifractal analysis of information processing in hippocampal neural ensembles during working memory under $\Delta 9$ tetrahydrocannabinol administration. Journal of Neuroscience Methods pii: S0165-0270(14)00264-7. doi: 10.1016/j.jneumeth.2014.07.013 
11) Fournier J, Müller CM, Schneider I, Laurent G. 2018. Spatial Information in a Non-retinotopic Visual Cortex. Neuron, 3;97(1):164-180.e7. doi: 10.1016/j.neuron.2017.11.017.

12) Frankel T. 2011. The Geometry of Physics: An Introduction. Cambridge University Press; IIIrd Ed. ISBN13: 978-1107602601

13) Friston K. 2010. The free-energy principle: a unified brain theory? Nat Rev Neurosci 11(2):127-138.

14) Geroch, Kronheimer, Penrose, 1972. Ideal points in space-time. Proc. Royal Soc. London A. 327, 545567.

15) Gold, D. A.; Runnegar, B.; Gehling, J. G.; Jacobs, D. K. (2015). Ancestral state reconstruction of ontogeny supports a bilaterian affinity for Dickinsonia". Evolution \& Development. 17 (6): 315-397. doi:10.1111/ede. 12168

16) Gorban AN; Karlin IV. 2016. Beyond Navier-Stokes equations: capillarity of ideal gas. Contemporary Physics, 58 (1): 70-90.

17) Ghrist R. 2014. Elementary Applied Topology, Amazon, ISBN: 978-1-5028-8085-1.

18) Heidegger M. 1957. Identity and difference. Harper \& Row, Publishers (New York, Evanston, and London).

19) Hoekzema, Renee S.; Brasier, Martin D.; Dunn, Frances S.; Liu, Alexander G. (2017-09-13). "Quantitative study of developmental biology confirms Dickinsonia as a metazoan". Proc. R. Soc. B. 284 (1862): 20171348. doi:10.1098/rspb.2017.1348

20) Hofstadter D, Sander E. 2011. Surfaces and Essences: Analogy as the Fuel and Fire of Thinking. Basic Books.

21) Holdeman JT. 2010. A Hermite finite element method for incompressible fluid flow. Int. J. Numer. Meth. Fluids, 64 (4): 376-408.

22) Hopf, H. 2001. Collected papers/Gesammelte Abhandlungen. Springer-Verlag, Berlin \& New York.

23) Ivantsov AY. 2007. Small Vendian transversely Articulated fossils. Paleontological Journal. 41 (2): $113-$ 122. doi:10.1134/S0031030107020013.

24) Johnson N. 2018, A visualization of the Hopf fibration. https://nilesjohnson.net/hopf.html (retrieved in 2018)

25) Johnstone PT. 1983. The point of pointless topology. Bulletin (New Series) of the American Mathematical Society. 8 (1): 41-53.

26) Koczkodaj WW, Magnot J-P, Mazurek J, Peters JF, Rakhshani H, Soltys M, Strzałka D, Szybowski J, Tozzi A. 2017. On normalization of inconsistency indicators in pairwise comparisons. International Journal of Approximate Reasoning.86, 73-79. http://doi.org/10.1016/j.ijar.2017.04.005.

27) Li H, Fang Q, Ge Y, Li Z, Meng J, Zhu J, Yu H. 2018. Relationship between the Dynamics of Orientation Tuning and Spatiotemporal Receptive Field Structures of Cat LGN Neurons. Neuroscience. 2018 May 1;377:26-39. doi: 10.1016/j.neuroscience.2018.02.024.

28) Mandelbrot B. 1967. How Long Is the Coast of Britain?. Science. 156 (3775): 636-638. doi:10.1126/science.156.3775.636

29) Mazade R, Alonso JM. 2017. Thalamocortical processing in vision. Vis Neurosci. 2017 Jan;34:E007. doi: $10.1017 / \mathrm{S} 0952523817000049$.

30) Munkres JR. 2000. Topology, $2^{\text {nd }}$ Ed. Prentice-Hall, Upper Saddle River, NJ, MR0464128.

31) Nagel T. 1974. What Is It Like to Be a Bat?. The Philosophical Review. 83 (4): 435-450. doi:10.2307/2183914

32) Pavese F, Charki A. 2016. Some important features of the proposed new definition of the International System of Units (SI): realization and hierarchical problems that the users should know about. Int.J.Metrol.Qual.Eng.7,403. DOI:10.1051/ijmqe/2016023.

33) Peters JF. 2016a. Computational Proximity. Excursions in the Topology of Digital Images. Edited by Intelligent Systems Reference Library. Berlin: Springer-Verlag, , Switzerland. doi:10.1007/978-3-31930262-1.

34) Peters JF, Ramanna S, Tozzi A, İnan E. 2017. Bold-Independent Computational Entropy Assesses Functional Donut-Like Structures in Brain fMRI Images. Front Hum Neurosci. 2017 Feb 1;11:38. doi: 10.3389/fnhum.2017.00038. eCollection 2017.

35) Popkin RH, Maia Neto JR (Editors). 2007. Skepticism: An Anthology. Prometheus Books. ISBN 1591024749 (ISBN13: 9781591024743 ). 
36) Sengupta B, Tozzi A, Coray GK, Douglas PK, Friston KJ. 2016. Towards a Neuronal Gauge Theory. PLOS Biology 14 (3): e1002400. doi:10.1371/journal.pbio.1002400.

37) Sowa JF; Majumdar AK. 2003. Analogicalal reasoning. Conceptual Structures for Knowledge Creation and Communication, Proceedings of ICCS 2003. Berlin: Springer-Verlag.

38) Tozzi A, Peters JF. 2016. Towards a Fourth Spatial Dimension of Brain Activity. Cognitive Neurodynamics 10 (3): 189-199. doi:10.1007/s11571-016-9379-z.

39) Tozzi A, Peters JF. 2018. What it is like to be "the same"? Progress in Biophysics and Molecular Biology. 133, 30-35. https://doi.org/10.1016/j.pbiomolbio.2017.10.005.

40) Tozzi A, Peters JF, Jaušovec N. 2018a. EEG dynamics on hyperbolic manifolds. Neurosci Lett. https://doi.org/10.1016/j.neulet.2018.07.035.

41) Tozzi A, Peters JF, Chafin C, De Falco D, Today J. 2018b. A timeless biology. Progr Biophys Mol Biol. 134, 38-43. doi: 10.1016/j.pbiomolbio.2017.12.002.

42) Van Hooser SD, Heimel JA, Nelson SB. 2005. Functional cell classes and functional architecture in the early visual system of a highly visual rodent. Prog Brain Res. 149:127-45.

43) Vankerschaver J, Leok M. 2014. A novel formulation of point vortex dynamics on the sphere: geometrical and numerical aspects. J. Nonlin. Sci. 24 (1): 1-37.

44) Viswanathan P, Nieder A. 2017. Visual Receptive Field Heterogeneity and Functional Connectivity of Adjacent Neurons in Primate Frontoparietal Association Cortices. J Neurosci. 2017 Sep 13;37(37):89198928. doi: 10.1523/JNEUROSCI.0829-17.2017. Epub 2017 Aug 11.

45) Wang BQ, Wang Gm Zhou X-H. 2015. A local wavelet transform on the torus T2. International Journal of Wavelets, Multiresolution and Information Processing. $13 \quad$ (04): 1550027. https://doi.org/10.1142/S0219691315500277

46) White FM. 2006. Viscous Fluid Flow, McGraw-Hill, ISBN 978-0-07-124493-0

47) Wigner E. 1960. The Unreasonable Effectiveness of Mathematics in the Natural Sciences. In: Communications in Pure and Applied Mathematics, vol. 13, No. I. New York: John Wiley \& Sons, Inc. 